\section{Utilização de medicamentos por idosos brasileiros, de acordo com a faixa etária: um inquérito postal}

\author{
Use of medications by elderly Brazilians according \\ to age: a postal survey
}

\author{
1 Faculdade de Farmácia, \\ Universidade Federal de \\ Minas Gerais, Belo Horizonte, \\ Brasil. \\ 2 Departamento de Nutrição \\ e Saúde, Universidade \\ Federal de Viçosa, Viçosa \\ Brasil. \\ 3 Escola Nacional de Saúde \\ Pública Sergio Arouca, \\ Fundação Oswaldo Cruz, Rio \\ de Janeiro, Brasil. \\ Correspondência \\ A. L. Silva \\ Departamento de Farmácia \\ Social, Faculdade de \\ Farmácia, Universidade \\ Federal de Minas Gerais. \\ Rua Flor de Índio 100, bloco \\ C, apto. 301, Belo Horizonte, \\ MG 31270-215, Brasil. \\ andyfarmacia@hotmail.com
}

\section{Abstract}

The objective of this study was to evaluate factors associated with use of medicines by the elderly. A national postal survey was conducted with a random sample of 3,000 elderly individuals selected from the registry of the Brazilian National Social Security Institute. The study estimated the prevalence and average number of medicines used in the 15 days prior to the survey, according to age bracket. Prevalence of use of medication was $83.0 \%: 78.8 \%$ in the 60-69 year bracket and $87.3 \%$ in individuals 70 years or older $(p<0.05)$. Older elders had taken an average of 4.4 drugs, as compared to 3.3 among younger elders. Drugs for the cardiovascular system were the most widely used. Age 70 years and older, female gender, poor self-rated health, interruption of routine daily activities, six or more medical visits in the previous year, private health insurance, and reporting of four or more illnesses were independently associated with use of medications $(p<0.05)$. The results expand the knowledge on use of medications among elderly Brazilians, emphasizing the need to improve pharmaceutical care focused on this subgroup of the population.

Pharmacoepidemiology; Polypharmacy; Drug Utilization; Aged
Anderson Lourenço da Silva 1

Andréia Queiroz Ribeiro 2

Carlos Henrique Klein 3

Francisco de Assis Acurcio 1

\section{Introdução}

Nos últimos anos, a população idosa brasileira apresentou um incremento quase duas vezes superior ao da população geral, sendo o segmento populacional que mais tem aumentado. Em 2007, a Pesquisa Nacional por Amostra de Domicílios (PNAD) contabilizou a existência de quase 20 milhões de idosos no Brasil, correspondendo a 10,5\% do total da população ${ }^{1}$. Segundo estimativas do Instituto Brasileiro de Geografia e Estatística (IBGE), em 2030 os idosos representarão aproximadamente $19 \%$ da população brasileira 2. Uma das consequências da longevidade será o aumento das demandas por atenção e cuidado, uma vez que os idosos apresentam maior incidência de doenças crônicas, pior capacidade funcional e menor autonomia 3,4 .

Nesse cenário de envelhecimento populacional e de maior preocupação em relação à saúde do idoso, os estudos farmacoepidemiológicos têm ganhado cada vez mais importância. No Brasil, a utilização de grande número de medicamentos é amplamente observada entre indivíduos com 60 anos ou mais 5,6,7. Além dos fatores clínicos que fazem com que os idosos necessitem de farmacoterapia, outros fatores podem estar associados ao uso demasiado de medicamentos, sendo um dos principais a ideia impregnada na sociedade de que a única possibilidade de se ter saúde é consumir saúde. Isso 
implica consumir medicamentos, importante símbolo de saúde nesta sociedade ${ }^{8}$. Sendo assim, identificar as características e os fatores associados ao consumo de medicamentos pelos idosos brasileiros pode auxiliar no planejamento de ações para promoção do uso racional de medicamentos e, consequentemente, favorecer uma melhor qualidade de vida para este grupo etário, além de contribuir para a diminuição de gastos desnecessários com estas tecnologias pelo sistema de saúde.

Observa-se um número crescente de estudos farmacoepidemiológicos do envelhecimento no Brasil que abordam diferentes aspectos, tais como os fatores associados ao uso de medicamentos, a polifarmácia, a automedicação, o alto custo dos medicamentos, seu uso inadequado, as reações adversas aos medicamentos, as interações medicamentosas, bem como os problemas de complexidade das prescrições 9,10,11,12,13,14,15,16. Embora haja consenso de que o uso de medicamentos aumenta com a idade, ainda se registra uma lacuna no conhecimento dos diferenciais etários na farmacoterapia por idosos brasileiros, apesar de sua importância para a melhor compreensão do perfil de utilização de medicamentos neste grupo etário.

O objetivo deste estudo foi caracterizar o uso de medicamentos entre idosos beneficiários do Instituto Nacional do Seguro Social (INSS) e identificar os fatores associados a este uso, focalizando as diferenças entre faixas etárias.

\section{Métodos}

Delineamento, população-alvo e amostra

O presente estudo integra o projeto multicêntrico Perfil de Utilização de Medicamentos por Aposentados Brasileiros, desenvolvido em nível nacional sob a coordenação da Faculdade de Farmácia da Universidade Federal de Minas Gerais (UFMG) e da Escola Nacional de Saúde Pública Sergio Arouca da Fundação Oswaldo Cruz (Fiocruz). A estratégia geral de delineamento foi a de um estudo epidemiológico seccional (inquérito), com abordagens postal (questionários de autopreenchimento) e domiciliar (questionários preenchidos por entrevistadores). As coletas de informações foram feitas pela abordagem postal em três amostras: no Brasil e nos municípios de Belo Horizonte e Rio de Janeiro. As informações diretamente nos domicílios foram feitas em duas amostras: nos municípios de Belo Horizonte e do Rio de Janeiro. Maiores detalhes referentes à metodologia do projeto podem ser obtidos em Acurcio et al. 17 .
Este estudo enfocará, especificamente, as informações obtidas pelo inquérito postal nacional.

A população-alvo deste trabalho foi constituída por beneficiários vinculados ao Regime Geral de Previdência Social/INSS/MPAS, com 60 anos ou mais de idade. Os participantes foram selecionados aleatoriamente com base no cadastro de aposentados e pensionistas do Ministério da Previdência e Assistência Social, de forma a constituírem uma amostra representativa da população-alvo.

Em 14 de dezembro de 2002, o cadastro da Empresa de Processamento de Dados da Previdência Social (DATAPREV) registrava 10.446.986 brasileiros, com idade igual ou superior a 60 anos, que recebiam benefícios (aposentadoria, pensão ou amparo social ao idoso) previdenciários e assistenciais ativos de prestação continuada pela Previdência Social. A seleção dos participantes foi realizada pela DATAPREV, baseando-se em seu cadastro de beneficiários, por amostragem aleatória simples, em novembro de 2002. Para a pesquisa em nível nacional foram sorteados 3 mil indivíduos para receberem o questionário por via postal. Para a análise dos dados obtidos no inquérito postal nacional, assumiu-se a suposição de semelhança entre respondentes e não respondentes e, na expectativa de resposta por parte de um terço dos sorteados, os erros amostrais seriam de $3,1 \%$ a $0,6 \%$ para prevalências de $50 \%$ a $1 \%$, respectivamente, para um nível de $95 \%$ de confiança.

\section{Coleta dos dados}

Os questionários de autopreenchimento foram enviados duas vezes para os endereços dos aposentados sorteados, nos dias 17 de janeiro e 24 de fevereiro de 2003, com porte pago para a devolução da resposta. Foram aceitos questionários preenchidos até 31 de maio de 2003.

\section{Variáveis do estudo}

Os questionários constituíam-se em brochuras no tamanho A4, com 32 páginas, 21 das quais com questões. Eram compostos de perguntas fechadas e pré-codificadas, exceto aquelas relativas aos medicamentos utilizados. Esse questionário possibilitava a obtenção de informações referentes às características sociodemográficas, condições de saúde, uso de serviços de saúde e de medicamentos.

Para a realização das análises do presente artigo, foram classificados como medicamentos os produtos informados pelos participantes que se apresentavam na forma industrializada ou manipulada. Para a classificação dos produtos 
foram consultados os registros dos mesmos no site da Agência Nacional de Vigilância Sanitária (ANVISA).

Os dados sobre os medicamentos foram organizados em tabelas e sua identificação foi feita com o auxílio do Dicionário de Especialidades Farmacêuticas (DEF) 18 e do Anatomical Therapeutic Chemical Classification System (ATC) 19. Os princípios ativos dos medicamentos foram agrupados conforme preconiza essa classificação.

\section{Análise dos dados}

Para a realização da presente análise foram considerados apenas os dados dos idosos respondentes que informaram a idade/data de nascimento no questionário.

As unidades de análise foram os indivíduos e os medicamentos. A razão do número de remédios por entrevistado foi usada como indicador da intensidade de uso, calculada dividindo-se o número de medicamentos citados pelo número de entrevistados. A comparação por faixa etária considerou os grupos de idosos com 60-69 anos e com 70 anos ou mais.

As variáveis respostas foram uso de medicamentos e número de medicamentos utilizados, investigadas por meio da pergunta: "Nos últimos 15 dias, o(a) senhor(a) usou medicamentos?". As variáveis explicativas foram sexo, escolaridade, valor do benefício do INSS, percepção da própria saúde, restrição para realizar atividades habituais por problemas de saúde nas duas últimas semanas, ter estado acamado neste período, número de doenças autorreferidas, e uso de serviços de saúde (filiação a plano privado de saúde, número de consultas e de internações nos últimos 12 meses).

As diferenças entre as proporções foram testadas com o teste qui-quadrado de Pearson, e as diferenças entre médias com o teste t de Student. A análise de regressão de Poisson com variância robusta foi empregada para obter as estimativas das razões de prevalência e os respectivos intervalos de 95\% de confiança (IC95\%). As variáveis que na análise bivariada se associaram à variável dependente com nível de significância menor que 0,20 foram incluídas no modelo de Poisson multivariado. O modelo final foi obtido utilizando-se o método de "trás para frente", com teste de significância da eliminação da variável em cada estágio. As variáveis que se associaram à variável dependente com nível de significância menor que 0,05 permaneceram no modelo final. Para todos os testes, utilizou-se nível de significância de 5\%. O programa empregado nas análises estatísticas foi o SPSS 17.0 (SPSS Inc., Chicago, Estados Unidos).

\section{Aspectos éticos}

A pesquisa foi conduzida dentro dos padrões exigidos pela Declaração de Helsinki e aprovada pelos Comitês de Ética em Pesquisa da UFMG e da Fiocruz.

\section{Resultados}

Perfil sociodemográfico e de saúde da amostra

Dos 3 mil idosos sorteados, 1.025 (34,2\%) responderam ao inquérito postal, não sendo encontradas diferenças estatisticamente significantes entre respondentes e não respondentes para as variáveis sexo, idade, região de moradia e valor do benefício do INSS 17 .

Para o presente artigo foram incluídos na análise 985 idosos, que tiveram data de nascimento informada no questionário. Do total de idosos analisados, 59,1\% eram mulheres. A maioria dos idosos possuía até o primário incompleto $(64,5 \%)$, sendo que aproximadamente um quarto nunca havia estudado, e cerca de $71 \%$ recebiam valores de benefício iguais ou inferiores ao salário mínimo vigente à época do inquérito ( $\mathrm{R} \$ 240,00)$. Mais da metade dos respondentes $(54,7 \%)$ consideravam sua saúde regular, e $36,6 \%$ dos idosos afirmaram ter interrompido as atividades habituais por motivo de saúde nos 15 dias anteriores à pesquisa. Pouco mais de um quinto dos respondentes relatou ter estado acamado nesse mesmo período. Cerca de $40 \%$ dos idosos informaram ter realizado seis ou mais consultas médicas no último ano, e a ocorrência de pelo menos uma internação hospitalar no mesmo período foi mencionada por $26,4 \%$ dos indivíduos. Dos idosos, 27,6\% afirmaram possuir plano de saúde. O relato de pelo menos uma doença crônica foi de $95,3 \%$, sendo que $36,5 \%$ dos idosos relataram quatro ou mais enfermidades (Tabela 1). Dentre as doenças referidas pelos idosos, destacaram-se pressão alta (58,6\%), problemas de visão (58,6\%) e artrite, artrose ou reumatismo (40,5\%).

Observamos que a proporção de mulheres no grupo etário de 70 anos ou mais foi maior do que no de 60-69 anos, sendo a diferença estatisticamente significativa. Também verificou-se que a proporção de idosos que recebem menor valor de benefício do INSS foi significativamente maior no grupo de 70 anos ou mais. Além dessas características, constatou-se maior proporção de idosos que deixaram de realizar atividades habituais por motivos de saúde, que referiram 4 ou mais doenças e que praticaram polifarmá- 
Distribuição de variáveis sociodemográficas, condição de saúde e utilização de serviço de saúde dos idosos que responderam ao inquérito postal, segundo grupos etários. Brasil, 2003.

\begin{tabular}{|c|c|c|c|c|}
\hline Variáveis & $\begin{array}{l}\text { Total } \\
\text { n (\%) }\end{array}$ & $\begin{array}{c}\text { 60-69 anos } \\
n(\%)\end{array}$ & $\begin{array}{c}70 \text { anos ou }+ \\
n(\%)\end{array}$ & Valor de $p$ \\
\hline \multicolumn{5}{|l|}{ Sexo } \\
\hline Masculino & $402(40,9)$ & $219(44,2)$ & $183(37,4)$ & \\
\hline Feminino & $582(59,1)$ & $276(55,8)$ & $306(62,6)$ & 0,030 \\
\hline \multicolumn{5}{|l|}{ Escolaridade } \\
\hline Nunca estudou & $251(25,9)$ & $117(24,0)$ & $134(27,8)$ & \\
\hline Alfabetização/Primário & $374(38,6)$ & $190(38,9)$ & $184(38,2)$ & \\
\hline \multicolumn{5}{|l|}{ incompleto } \\
\hline Primário completo & $201(20,7)$ & $96(19,7)$ & $105(21,8)$ & \\
\hline Primeiro grau a terceiro grau & $144(14,8)$ & $85(17,4)$ & $59(12,2)$ & 0,098 \\
\hline \multicolumn{5}{|l|}{ Valor do benefício do INSS } \\
\hline \multicolumn{5}{|l|}{ (salários mínimos *) } \\
\hline Até 1 & $701(71,3)$ & $335(68,0)$ & $366(74,7)$ & \\
\hline$<1$ & $282(28,7)$ & $158(32,0)$ & $124(25,3)$ & 0,019 \\
\hline \multicolumn{5}{|l|}{ Percepção da própria saúde } \\
\hline Muito boa/Boa & $254(25,9)$ & $131(26,6)$ & $123(25,3)$ & \\
\hline Regular & $536(54,7)$ & $274(55,6)$ & $262(53,8)$ & \\
\hline Ruim/Muito ruim & $190(19,4)$ & $88(17,8)$ & $102(20,9)$ & 0,469 \\
\hline \multicolumn{5}{|l|}{ Deixar de realizar atividades } \\
\hline \multicolumn{5}{|l|}{ habituais nos últimos 15 dias } \\
\hline Sim & $356(36,6)$ & $161(32,7)$ & $195(40,5)$ & \\
\hline Não & $618(63,4)$ & $332(67,3)$ & $286(59,5)$ & 0,011 \\
\hline \multicolumn{5}{|l|}{ Acamados nos últimos 15 dias } \\
\hline Sim & $208(21,4)$ & $100(20,4)$ & $108(22,4)$ & \\
\hline Não & $765(78,6)$ & $391(79,6)$ & $374(77,6)$ & 0,438 \\
\hline \multicolumn{5}{|l|}{ Consultas médicas no último ano } \\
\hline Nenhuma & $82(8,4)$ & $41(8,3)$ & $41(8,4)$ & \\
\hline $1-5$ & $511(52,0)$ & $271(54,7)$ & $240(49,3)$ & \\
\hline 6 ou mais & $389(39,6)$ & $183(37,0)$ & $206(42,3)$ & 0,204 \\
\hline \multicolumn{5}{|l|}{ Internações hospitalares no } \\
\hline \multicolumn{5}{|l|}{ último ano } \\
\hline Nenhuma & $714(73,6)$ & $372(75,9)$ & $342(71,2)$ & \\
\hline 1 ou mais & $256(26,4)$ & $118(24,1)$ & $138(28,8)$ & 0,099 \\
\hline \multicolumn{5}{|l|}{ Plano privado de saúde } \\
\hline Sim & $270(27,6)$ & $123(25,0)$ & $147(30,3)$ & \\
\hline Não & $707(72,4)$ & $369(75,0)$ & $338(69,7)$ & 0,064 \\
\hline \multicolumn{5}{|l|}{ Número de doenças } \\
\hline Nenhuma & $44(4,5)$ & $25(5,1)$ & $19(3,9)$ & \\
\hline $1-3$ & $581(59,0)$ & $315(63,6)$ & $266(54,3)$ & \\
\hline 4 ou mais & $360(36,5)$ & $155(31,3)$ & $205(41,8)$ & 0,003 \\
\hline \multicolumn{5}{|l|}{ Uso de medicamentos nos últimos } \\
\hline \multicolumn{5}{|l|}{15 dias } \\
\hline Nenhum & $167(17,0)$ & $105(21,2)$ & $62(12,7)$ & \\
\hline $1-4$ & $469(47,6)$ & $250(50,5)$ & $219(44,7)$ & \\
\hline 5 ou mais & $349(35,4)$ & $140(28,3)$ & $209(42,7)$ & $<0,001$ \\
\hline
\end{tabular}

INSS: Instituto Nacional do Seguro Social.

* $R \$ 240,00$ no ano de 2003 (época do estudo). 
cia (uso de 5 ou mais medicamentos) no grupo de 70 anos ou mais em comparação aos idosos mais jovens, sendo as diferenças estatisticamente significativas.

\section{Perfil de utilização de medicamentos}

A prevalência de uso de medicamentos nos 15 dias anteriores à resposta ao questionário foi de $83,0 \%$ e a de polifarmácia ocorreu em $35,4 \%$. Considerando a estratificação etária, foram observadas maiores prevalências de uso de medicamentos e de polifarmácia na faixa etária de 70 anos ou mais $(87,3 \%$ e $42,7 \%$, respectivamente) em comparação ao grupo de $60-69$ anos (78,8\% e $28,3 \%$, respectivamente), sendo estas diferenças estatisticamente significativas $(\mathrm{p}<0,001)$.

Os idosos utilizaram o total de 3.778 medicamentos, o que resultou em uma média de 3,8 $\pm 3,3$ medicamentos por pessoa. Essa média foi significativamente maior para os idosos da faixa etária mais elevada $(4,4 \pm 3,6)$ em comparação àqueles pertencentes à faixa etária mais jovem $(3,3 \pm 3,0)(\mathrm{p}<0,001)$.

Na Tabela 2 encontra-se a distribuição dos medicamentos de acordo com o primeiro e segundo níveis da classificação ATC. Dos medicamentos utilizados, 1.626 foram usados por idosos na faixa etária de 60-69 anos e 2.152 pelos idosos na faixa etária de 70 anos ou mais.

Os medicamentos mais utilizados pela amostra estudada e nas diferentes faixas etárias pertencem aos sistemas cardiovascular, nervoso e do trato alimentar/metabolismo. As razões de consumo de medicamentos pertencentes a esses três grupos, por indivíduo, foram maiores entre os idosos de maior idade em comparação aos idosos mais jovens: 1,32 vs. 1,02 para os cardiovasculares, 0,75 vs. 0,54 para os do sistema nervoso e 0,68 vs. 0,47 para os do trato alimentar e metabolismo, respectivamente.

Os subgrupos terapêuticos mais frequentemente usados no sistema cardiovascular foram diuréticos, agentes com ação no sistema renina-angiotensina e medicamentos para terapia cardíaca, correspondendo, respectivamente, às seguintes razões de uso: 0,33; 0,26 e 0,14. A diferença mais expressiva entre as faixas etárias foi observada para os medicamentos para terapia cardíaca, cujo consumo entre os idosos de maior idade foi $90 \%$ superior ao dos idosos mais jovens. Os subgrupos terapêuticos do sistema nervoso foram representados principalmente por analgésicos, psicolépticos e psicoanalépticos, cujo consumo foi superior entre os idosos da faixa etária de 70 anos ou mais $(25 \%, 41,7 \%$ e $88,9 \%$, respectivamente). Os medicamentos com ação no trato alimentar e metabolismo mais citados foram vitaminas, fármacos hipoglicemiantes, antiácidos e demais agentes para tratamento de úlcera péptica e flatulência. Os idosos de maior idade consumiram $66,7 \%$ mais vitaminas do que os mais jovens.

\section{Fatores associados ao uso de medicamentos}

Na análise univariada, sexo feminino, pior autopercepção de saúde, restrição das atividades habituais, estar acamado nos últimos 15 dias anteriores à pesquisa, maior número de consultas médicas, histórico de internação nos últimos 12 meses, participação em plano de saúde privado e maior número de doenças autorreferidas estavam associados de forma positiva e estatisticamente significativa ao uso de medicamento (Tabela 3). De modo geral, essas associações também foram observadas na análise estratificada por faixa etária. Exceções ocorreram dentre os idosos de 60-69 anos, na associação entre uso de medicamentos e autopercepção da saúde e participação em plano de saúde privado. Já dentre os idosos de 70 ou mais anos, associações sem significância estatística foram observadas entre uso de medicamentos e sexo, bem como percepção da saúde. Cabe ressaltar que a maior escolaridade se associou positiva e significativamente ao uso de medicamentos no grupo de idosos com 70 ou mais anos.

Considerando a amostra total, a média de remédios utilizados por idosos foi maior entre as mulheres, nos indivíduos de maior escolaridade, com pior percepção de saúde, maior número de doenças, que deixaram de realizar atividades habituais e que estiveram acamados nos 15 dias anteriores à pesquisa, idosos que realizaram maior número de consultas médicas, com histórico de internação nos últimos 12 meses e afiliados a plano privado de saúde $(\mathrm{p}<0,05)$. O mesmo foi observado ao se considerar as faixas etárias separadamente. A única exceção ocorreu no grupo dos idosos jovens em que a escolaridade não apresentou associação estatisticamente significativa com o número de medicamentos usados.

Os resultados da análise multivariada são apresentados na Tabela 4. Observou-se associação positiva e independente entre uso de medicamentos e faixa etária (70 ou mais anos), sexo (feminino), percepção de saúde (regular, ruim ou muito ruim), restrição de atividades habituais (sim), consultas médicas no último ano (6 ou mais), plano privado de saúde (sim) e número de doenças (4 ou mais). 
Tabela 2

Distribuição das frequências de uso e quantidade de medicamentos, por grupos e subgrupos anatomico-terapêuticos, por idosos que responderam ao inquérito postal, segundo grupos etários. Brasil, 2003 *.

\begin{tabular}{|c|c|c|c|c|c|c|}
\hline \multirow[t]{2}{*}{ Grupos anatômicos e terapêuticos } & \multicolumn{2}{|c|}{ Total ** } & \multicolumn{2}{|c|}{$60-69$ anos *** } & \multicolumn{2}{|c|}{70 ou mais anos \# } \\
\hline & n (\%) & $\begin{array}{l}\text { Medicamentos/ } \\
\text { idoso }\end{array}$ & n (\%) & $\begin{array}{l}\text { Medicamentos/ } \\
\text { idoso }\end{array}$ & $\mathrm{n}(\%)$ & $\begin{array}{l}\text { Medicamentos/ } \\
\text { idoso }\end{array}$ \\
\hline A: Trato alimentar/Metabolismo & $566(15,0)$ & 0,57 & $234(14,4)$ & 0,47 & $332(15,4)$ & 0,68 \\
\hline $\begin{array}{l}\text { A02: Antiácidos, fármacos para } \\
\text { tratamento de úlcera péptica e } \\
\text { flatulência }\end{array}$ & $118(3,1)$ & 0,12 & $44(2,7)$ & 0,09 & $74(3,4)$ & 0,15 \\
\hline $\begin{array}{l}\text { A03: Fármacos para tratamento } \\
\text { de desordens funcionais do trato } \\
\text { gastrintestinal }\end{array}$ & $24(0,6)$ & 0,02 & $11(0,7)$ & 0,02 & $13(0,6)$ & 0,03 \\
\hline A10: Hipoglicemiantes & $154(4,1)$ & 0,16 & $73(4,5)$ & 0,15 & $81(3,8)$ & 0,17 \\
\hline A11: Vitaminas & $159(4,2)$ & 0,16 & $59(3,6)$ & 0,12 & $100(4,6)$ & 0,20 \\
\hline A12: Suplementos minerais & $62(1,6)$ & 0,06 & $29(1,8)$ & 0,06 & $33(1,5)$ & 0,07 \\
\hline $\begin{array}{l}\text { B: Sangue e órgãos formadores de } \\
\text { sangue }\end{array}$ & $141(3,7)$ & 0,14 & $64(3,9)$ & 0,13 & $77(3,6)$ & 0,16 \\
\hline B01: Agentes trombolíticos & $93(2,5)$ & 0,09 & $41(2,5)$ & 0,08 & $52(2,4)$ & 0,11 \\
\hline B03: Antianêmicos & $43(1,1)$ & 0,04 & $22(1,3)$ & 0,04 & $21(1,0)$ & 0,04 \\
\hline C: Sistema cardiovascular & $1.150(30,4)$ & 1,17 & $503(30,9)$ & 1,02 & $647(30,1)$ & 1,32 \\
\hline C01: Terapia cardíaca & $139(3,7)$ & 0,14 & $48(3,0)$ & 0,10 & $91(4,2)$ & 0,19 \\
\hline C03: Diuréticos & $326(8,6)$ & 0,33 & $139(8,6)$ & 0,28 & $187(8,7)$ & 0,38 \\
\hline C07: Agentes $\beta$-bloqueadores & $128(3,4)$ & 0,13 & $64(3,9)$ & 0,13 & $64(3,0)$ & 0,13 \\
\hline $\begin{array}{l}\text { C08: Bloqueadores dos canais } \\
\text { de cálcio }\end{array}$ & $99(2,6)$ & 0,10 & $40(2,5)$ & 0,08 & $59(2,7)$ & 0,12 \\
\hline $\begin{array}{l}\text { C09: Agentes do sistema renina- } \\
\text { angiotensina }\end{array}$ & $261(6,9)$ & 0,26 & $128(7,9)$ & 0,26 & $133(6,2)$ & 0,27 \\
\hline C10: Hipolipemiantes & $75(2,0)$ & 0,08 & $33(2,0)$ & 0,07 & $42(2,0)$ & 0,09 \\
\hline D: Agentes dermatológicos & $115(3,1)$ & 0,12 & $60(3,7)$ & 0,12 & $55(2,6)$ & 0,11 \\
\hline D01: Antifúngicos & $18(0,5)$ & 0,02 & $11(0,7)$ & 0,02 & $7(0,3)$ & 0,01 \\
\hline $\begin{array}{l}\text { D02: Emolientes e protetores } \\
\text { dermatológicos }\end{array}$ & $3(0,1)$ & 0,00 & & 0,00 & $3(0,1)$ & 0,01 \\
\hline $\begin{array}{l}\text { D06: Antibióticos e quimioterá- } \\
\text { picos dermatológicos }\end{array}$ & $26(0,7)$ & 0,03 & $11(0,7)$ & 0,02 & $15(0,7)$ & 0,03 \\
\hline $\begin{array}{l}\text { D07: Corticóides, preparações } \\
\text { dermatológicas }\end{array}$ & $54(1,4)$ & 0,05 & $30(1,8)$ & 0,06 & $24(1,1)$ & 0,05 \\
\hline $\begin{array}{l}\text { G: Sistema geniturinário e } \\
\text { hormônios sexuais }\end{array}$ & $43(1,1)$ & 0,04 & $27(1,7)$ & 0,05 & $16(0,8)$ & 0,03 \\
\hline $\begin{array}{l}\text { G03: Hormônios sexuais e } \\
\text { moduladores do sistema genital }\end{array}$ & $31(0,8)$ & 0,03 & $22(1,3)$ & 0,04 & $9(0,5)$ & 0,02 \\
\hline G04: Fármacos urológicos & $9(0,2)$ & 0,01 & $3(0,2)$ & 0,01 & $6(0,3)$ & 0,01 \\
\hline $\begin{array}{l}\text { H: Preparações hormonais } \\
\text { sistêmicas }\end{array}$ & $55(1,5)$ & 0,06 & $23(1,4)$ & 0,05 & $32(1,5)$ & 0,07 \\
\hline $\begin{array}{l}\text { H02: Corticóides de uso } \\
\text { sistêmico }\end{array}$ & $33(0,9)$ & 0,03 & $13(0,8)$ & 0,03 & $20(1,0)$ & 0,04 \\
\hline H03: Terapia para tireoide & $19(0,5)$ & 0,02 & $8(0,5)$ & 0,02 & $11(0,5)$ & 0,02 \\
\hline $\begin{array}{l}\mathrm{J} \text { : Anti-infecciosos gerais de uso } \\
\text { sistêmico }\end{array}$ & $110(2,9)$ & 0,11 & $44(2,7)$ & 0,09 & $66(3,1)$ & 0,13 \\
\hline J01: Antibacterianos & $99(2,6)$ & 0,10 & $40(2,5)$ & 0,08 & $59(2,7)$ & 0,12 \\
\hline J02: Antimicóticos & $4(0,1)$ & 0,00 & $3(0,2)$ & 0,01 & $1(0,1)$ & 0,00 \\
\hline J05: Antivirais & $5(0,1)$ & 0,01 & $1(0,1)$ & 0,00 & $4(0,2)$ & 0,01 \\
\hline
\end{tabular}

(continua) 


\begin{tabular}{|c|c|c|c|c|c|c|}
\hline \multirow[t]{2}{*}{ Grupos anatômicos e terapêuticos } & \multicolumn{2}{|c|}{ Total ** } & \multicolumn{2}{|c|}{$60-69$ anos *** } & \multicolumn{2}{|c|}{70 ou mais anos \# } \\
\hline & n (\%) & $\begin{array}{l}\text { Medicamentos/ } \\
\text { idoso }\end{array}$ & n (\%) & $\begin{array}{l}\text { Medicamentos/ } \\
\text { idoso }\end{array}$ & n (\%) & \\
\hline $\begin{array}{l}\text { L: Antineoplásicos e } \\
\text { imunomoduladores }\end{array}$ & $5(0,1)$ & 0,01 & $3(0,2)$ & 0,01 & $2(0,1)$ & 0,00 \\
\hline L02: Terapia endócrina & $3(0,1)$ & 0,00 & $2(0,1)$ & 0,00 & $1(0,1)$ & 0,00 \\
\hline $\begin{array}{l}\text { M: Agentes do sistema musculo- } \\
\text { esquelético }\end{array}$ & $342(9,1)$ & 0,35 & $157(9,7)$ & 0,32 & $185(8,6)$ & 0,38 \\
\hline $\begin{array}{l}\text { M01: Antirreumáticos e anti- } \\
\text { inflamatórios }\end{array}$ & $277(7,3)$ & 0,28 & $133(8,2)$ & 0,27 & $144(6,7)$ & 0,29 \\
\hline M04: Antigotosos & $19(0,5)$ & 0,02 & $9(0,6)$ & 0,02 & $10(0,5)$ & 0,02 \\
\hline $\begin{array}{l}\text { M05: Medicamentos para } \\
\text { tratamento de doenças ósseas }\end{array}$ & $24(0,6)$ & 0,02 & $8(0,5)$ & 0,02 & $16(0,7)$ & 0,03 \\
\hline N: Sistema nervoso & $634(16,8)$ & 0,64 & $266(16,4)$ & 0,54 & $368(17,1)$ & 0,75 \\
\hline N02: Analgésicos & $222(5,9)$ & 0,23 & $101(6,2)$ & 0,20 & $121(5,6)$ & 0,25 \\
\hline N05: Psicolépticos & $139(3,7)$ & 0,14 & $58(3,6)$ & 0,12 & $81(3,8)$ & 0,17 \\
\hline N06: Psicoanalépticos & $129(3,4)$ & 0,13 & $47(2,9)$ & 0,09 & $82(3,8)$ & 0,17 \\
\hline $\begin{array}{l}\text { N07: Outros medicamentos do } \\
\text { sistema nervoso }\end{array}$ & $79(2,1)$ & 0,08 & $27(1,7)$ & 0,05 & $52(2,4)$ & 0,11 \\
\hline P: Agentes antiparasitários & $18(0,5)$ & 0,02 & $8(0,5)$ & 0,02 & $10(0,5)$ & 0,02 \\
\hline P01: Antiprotozoários & $12(0,3)$ & 0,01 & $5(0,3)$ & 0,01 & $7(0,3)$ & 0,01 \\
\hline P02: Anti-helmínticos & $5(0,1)$ & 0,01 & $2(0,1)$ & 0,00 & $3(0,1)$ & 0,01 \\
\hline R: Sistema respiratório & $138(3,6)$ & 0,14 & $52(3,2)$ & 0,11 & $86(4,0)$ & 0,18 \\
\hline R01: Preparações nasais & $15(0,4)$ & 0,02 & $8(0,5)$ & 0,02 & $7(0,3)$ & 0,01 \\
\hline R03: Antiasmáticos & $62(1,6)$ & 0,06 & $21(1,3)$ & 0,04 & $41(1,9)$ & 0,08 \\
\hline $\begin{array}{l}\text { R05: Preparados contra a tosse e } \\
\text { resfriados }\end{array}$ & $35(0,9)$ & 0,04 & $9(0,6)$ & 0,02 & $26(1,2)$ & 0,05 \\
\hline $\begin{array}{l}\text { R06: Anti-histamínicos de uso } \\
\text { sistêmico }\end{array}$ & $25(0,7)$ & 0,03 & $13(0,8)$ & 0,03 & $12(0,6)$ & 0,02 \\
\hline S: Órgãos do sentido & $134(3,5)$ & 0,14 & $46(2,8)$ & 0,09 & $88(4,1)$ & 0,18 \\
\hline S01: Oftalmológicos & $127(3,4)$ & 0,13 & $44(2,7)$ & 0,09 & $83(3,9)$ & 0,17 \\
\hline Sem classificação ATC & $327(8,7)$ & 0,33 & $139(8,5)$ & 0,28 & $188(8,7)$ & 0,38 \\
\hline Total & $3.778(100,0)$ & 3,84 & $1.626(100,0)$ & 3,28 & $2.152(100,0)$ & 4,40 \\
\hline
\end{tabular}

ATC: Anatomical Therapeutic Chemical Classification System ${ }^{19}$

* São apresentados os subgrupos terapêuticos mais frequentes, ou seja, os que totalizam até 90,0\% dentro de cada nível;

** Total: número de medicamentos $=3.778$; número de usuários $=985$;

*** 60-69 anos: número de medicamentos $=1.629$; número de usuários $=495$;

\# 70 ou mais anos: número de medicamentos = 2.152; número de usuários $=490$.

\section{Discussão}

A realização de estudos abrangentes sobre a utilização de medicamentos por idosos, como o presente trabalho, constitui uma demanda importante em nosso país, uma vez que este tema ainda é insuficientemente abordado e os estudos realizados até o momento são restritos a alguns municípios brasileiros 9,12,13,14,15,20,21, o que geralmente impossibilita a extrapolação dos resultados para outras regiões e para o país. $\mathrm{O}$ presente trabalho adquire relevância por ter sido desenhado de modo a propiciar uma visão ampla do uso de medicamentos pelos idosos brasileiros, visto que a grande maioria destes indivíduos está vinculada ao INSS. No entanto, é importante mencionar que o tamanho da amostra não foi planejado para testar diferenças de idade, portanto, algumas das diferenças podem não ter sido detectadas por insuficiência da quantidade de observações. Por outro lado, não é razoável supor que esse fato tenha comprometido as diferenças observadas e descritas. Ressalta-se, ainda, que as estimativas obtidas 
Tabela 3

Prevalências e razões de prevalência de uso e quantidades médias de medicamentos utilizados pelos idosos, por variáveis sociodemográficas e de saúde dos idosos que responderam ao inquérito postal, segundo grupos etários. Brasil, 2003.

\begin{tabular}{|c|c|c|c|c|c|c|c|c|c|}
\hline \multirow{2}{*}{$\begin{array}{l}\text { Características } \\
\text { sociodemográficas }\end{array}$} & \multicolumn{3}{|c|}{ Total } & \multicolumn{3}{|c|}{ 60-69 anos } & \multicolumn{3}{|c|}{70 ou mais anos } \\
\hline & $\begin{array}{l}\text { Uso } \\
n(\%)\end{array}$ & RP (IC95\%) & Média (DP) & $\begin{array}{l}\text { Uso } \\
\text { n (\%) }\end{array}$ & RP (IC95\%) & Média (DP) & $\begin{array}{l}\text { Uso } \\
\text { n (\%) }\end{array}$ & RP (IC95\%) & Média (DP) \\
\hline \multicolumn{10}{|l|}{ Sexo } \\
\hline Masculino & $303(75,4)$ & 1,00 & $3,30(3,25)$ & $151(68,9)$ & 1,00 & $2,60(2,77)$ & $152(83,1)$ & 1,00 & $4,14(3,57)$ \\
\hline Feminino & $514(88,3)$ & $\begin{array}{c}1,17 \\
(1,10-1,25)\end{array}$ & $4,21(3,36)$ & $239(86,6)$ & $1,26(1,14-1,40)$ & $3,83(3,04)$ & $275(89,9)$ & $1,08(1,00-1,17)$ & $4,55(3,59)$ \\
\hline Valor de $p$ & & & $<0,001$ & & & $<0,001$ & & & 0,222 \\
\hline \multicolumn{10}{|l|}{ Escolaridade } \\
\hline $\begin{array}{l}\text { Até Primeiro Grau } \\
\text { incompleto }\end{array}$ & $686(83,1)$ & 1,00 & $3,75(3,28)$ & $318(78,9)$ & 1,00 & $3,23(2,99)$ & $368(87,0)$ & 1,00 & $4,24(3,47)$ \\
\hline $\begin{array}{l}\text { Primeiro Grau } \\
\text { completo ou mais }\end{array}$ & $124(86,1)$ & $\begin{array}{c}1,04 \\
(0,96-1,12)\end{array}$ & $4,48(3,55)$ & $67(78,8)$ & $0,99(0,88-1,13)$ & $3,51(2,81)$ & $57(96,6)$ & $1,11(1,05-1,18)$ & $5,88(4,03)$ \\
\hline Valor de $p$ & & & 0,015 & & & 0,437 & & & 0,001 \\
\hline \multicolumn{10}{|l|}{$\begin{array}{l}\text { Valor do benefício em } \\
\text { salários mínimos }\end{array}$} \\
\hline Até 1 & $581(82,9)$ & 1,00 & $3,79(3,31)$ & $263(78,5)$ & 1,00 & $3,31(3,09)$ & $318(86,9)$ & 1,00 & $4,23(3,45)$ \\
\hline$<1$ & $235(83,3)$ & $\begin{array}{c}1,00 \\
(0,95-1,07)\end{array}$ & $3,95(3,41)$ & $125(79,1)$ & $0,96(0,61-1,53)$ & $3,24(2,76)$ & $110(88,7)$ & $0,84(0,45-1,59)$ & $4,86(3,92)$ \\
\hline Valor de $p$ & & & 0,492 & & & 0,801 & & & 0,090 \\
\hline \multicolumn{10}{|l|}{$\begin{array}{l}\text { Percepção da própria } \\
\text { saúde }\end{array}$} \\
\hline Muito boa/Boa & $179(70,5)$ & 1,00 & $2,54(2,80)$ & $81(61,8)$ & 1,00 & $2,05(2,41)$ & $98(79,7)$ & 1,00 & $3,06(3,10)$ \\
\hline Regular & $465(86,8)$ & $\begin{array}{c}1,23 \\
(1,04-1,46)\end{array}$ & $3,96(3,20)$ & $233(85,0)$ & $1,36(1,06-1,75)$ & $3,47(2,78)$ & $232(88,5)$ & $1,11(0,88-1,41)$ & $4,47(3,52)$ \\
\hline Ruim/Muito ruim & $171(90,0)$ & $\begin{array}{c}1,28 \\
(1,04-1,57)\end{array}$ & $5,27(3,74)$ & $74(84,1)$ & $1,34(0,98-1,84)$ & $4,51(3,68)$ & $97(95,1)$ & $1,19(0,90-1,58)$ & $5,92(3,68)$ \\
\hline Valor de p & & & $<0,001$ & & & 0,001 & & & 0,001 \\
\hline \multicolumn{10}{|l|}{$\begin{array}{l}\text { Deixar de realizar } \\
\text { atividades habituais }\end{array}$} \\
\hline Não & $480(77,7)$ & 1,00 & $3,03(2,89)$ & $244(73,5)$ & 1,00 & $2,70(2,67)$ & $236(82,5)$ & 1,00 & $3,41(3,08)$ \\
\hline $\operatorname{Sim}$ & $332(93,3)$ & $\begin{array}{c}1,20 \\
(1,14-1,26)\end{array}$ & $5,30(3,57)$ & $145(90,1)$ & $1,23(1,13-1,33)$ & $4,48(3,19)$ & $187(95,9)$ & $1,16(1,09-1,24)$ & $5,97(3,73)$ \\
\hline $\begin{array}{l}\text { Valor de } p \\
\text { Acamados nos } \\
\text { últimos } 15 \text { dias }\end{array}$ & & & $<0,001$ & & & $<0,001$ & & & $<0,001$ \\
\hline Não & $616(80,5)$ & 1,00 & $3,42(3,09)$ & $298(76,2)$ & 1,00 & $2,98(2,84)$ & $318(85,0)$ & 1,00 & $3,87(3,27)$ \\
\hline Sim & $195(93,8)$ & $\begin{array}{c}1,16 \\
(1,11-1,22)\end{array}$ & $5,40(3,70)$ & $90(90,0)$ & $1,18(1,08-1,29)$ & $4,44(3,09)$ & $105(97,2)$ & $1,14(1,08-1,21)$ & $6,30(3,99)$ \\
\hline Valor de $p$ & & & $<0,001$ & & & $<0,001$ & & & $<0,001$ \\
\hline
\end{tabular}

(continua) 
Prevalências e razões de prevalência de uso e quantidades médias de medicamentos utilizados pelos idosos, por variáveis sociodemográficas e de saúde dos idosos que responderam ao inquérito postal, segundo grupos etários. Brasil, 2003.

\begin{tabular}{|c|c|c|c|c|c|c|c|c|c|}
\hline \multirow{2}{*}{$\begin{array}{l}\text { Características } \\
\text { sociodemográficas }\end{array}$} & \multicolumn{3}{|c|}{ Total } & \multicolumn{3}{|c|}{ 60-69 anos } & \multicolumn{3}{|c|}{70 ou mais anos } \\
\hline & $\begin{array}{l}\text { Uso } \\
\text { n (\%) }\end{array}$ & RP (IC95\%) & Média (DP) & $\begin{array}{l}\text { Uso } \\
\text { n (\%) }\end{array}$ & RP (IC95\%) & Média (DP) & $\begin{array}{l}\text { Uso } \\
\text { n (\%) }\end{array}$ & RP (IC95\%) & $\begin{array}{l}\text { Média } \\
\text { (DP) }\end{array}$ \\
\hline \multicolumn{10}{|c|}{$\begin{array}{l}\text { Consultas médicas no } \\
\text { último ano }\end{array}$} \\
\hline Até 5 & $447(75,4)$ & 1,00 & $2,91(2,94)$ & $216(69,2)$ & 1,00 & $2,43(2,64)$ & $231(82,2)$ & 1,00 & $3,44(3,16)$ \\
\hline 6 ou mais & $370(95,1)$ & $\begin{array}{c}1,26 \\
(1,20-1,33)\end{array}$ & $5,28(3,41)$ & $174(95,1)$ & $1,37(1,27-1,49)$ & $4,74(2,98)$ & $196(95,1)$ & $1,16(1,09-1,23)$ & $5,75(3,69)$ \\
\hline Valor de $p$ & & & $<0,001$ & & & $<0,001$ & & & $<0,001$ \\
\hline \multicolumn{10}{|l|}{ no último ano } \\
\hline Nenhuma & $575(80,5)$ & 1,00 & $3,46(3,08)$ & $281(75,5)$ & 1,00 & $3,05(2,90)$ & $294(86,0)$ & 1,00 & $3,90(3,21)$ \\
\hline 1 ou mais & $230(89,8)$ & $\begin{array}{c}1,12 \\
(1,06-1,18)\end{array}$ & $4,90(3,76)$ & $104(88,1)$ & $1,17(1,07-1,27)$ & $3,97(3,04)$ & $126(91,3)$ & $1,06(0,99-1,14)$ & $5,69(4,14)$ \\
\hline Valor de p & & & $<0,001$ & & & 0,003 & & & $<0,001$ \\
\hline \multicolumn{10}{|l|}{ Plano de saúde } \\
\hline Não & $574(81,2)$ & 1,00 & $3,56(3,19)$ & $288(78,0)$ & 1,00 & $3,10(2,88)$ & $286(84,6)$ & 1,00 & $4,07(3,42)$ \\
\hline $\operatorname{Sim}$ & $239(88,5)$ & $\begin{array}{c}1,09 \\
(1,03-1,15)\end{array}$ & $4,60(3,62)$ & $100(81,3)$ & $1,04(0,94-1,15)$ & $3,89(3,21)$ & $139(94,6)$ & $1,12(1,05-1,19)$ & $5,20(3,83)$ \\
\hline Valor de $p$ & & & $<0,001$ & & & $<0,001$ & & & 0,001 \\
\hline \multicolumn{10}{|l|}{ Número de doenças } \\
\hline Até 3 & $483(77,3)$ & 1,00 & $2,94(2,92)$ & $245(72,1)$ & 1,00 & $2,48(2,52)$ & $238(83,5)$ & 1,00 & $3,49(3,25)$ \\
\hline 4 ou mais & $335(93,1)$ & $\begin{array}{c}1,20 \\
(1,14-1,27)\end{array}$ & $5,39(3,45)$ & $145(93,5)$ & $1,30(1,20-1,40)$ & $5,06(3,15)$ & $190(92,7)$ & $1,11(1,04-1,18)$ & $5,64(3,65)$ \\
\hline Valor de $p$ & & & $<0,001$ & & & $<0,001$ & & & $<0,001$ \\
\hline
\end{tabular}

IC95\%: intervalo de 95\% de confiança; RP: razão de prevalência.

são generalizáveis apenas para aposentados e pensionistas do INSS, com idade igual ou superior a 60 anos. Portanto, a extrapolação dos resultados para o conjunto dos idosos brasileiros requer cautela.

Outro aspecto relevante refere-se à baixa cobertura de respostas ao inquérito postal, o que não significa necessariamente baixa representatividade. Os atributos disponíveis nas informações cedidas pela DATAPREV para este inquérito (sexo, idade, região de residência e valor do benefício na época da pesquisa) foram utilizados na análise de viés de não-resposta. Verificou-se semelhanças de proporções para cada um desses atributos entre os respondentes e os não respondentes. Se esse achado não permite concluir que a amostra dos que responderam pode ser de fato representativa do total, também não permite afastar esta hipótese 17 .
A amostra foi composta predominantemente de mulheres, por idosos com baixa escolaridade e recebendo baixos valores de benefícios do INSS, o que mostra coerência com a situação da população idosa brasileira segundo dados do IBGE 1. A maior proporção de mulheres entre os idosos é uma característica marcante do envelhecimento populacional no Brasil e no mundo, sendo que o predomínio do sexo feminino é maior nas idades mais avançadas 1, fenômeno também verificado em nosso estudo. É preocupante observar que mais da metade dos participantes não tinha o primário completo, sendo que um quarto deles nunca estudou. A baixa escolaridade influi na compreensão e cumprimento da prescrição, podendo resultar em troca de medicamentos e outros erros na sua utilização.

A prevalência de uso de medicamentos foi elevada (83\%) assim como a média de produtos 
Tabela 4

Resultados finais da análise multivariada da associação entre as condições sociodemográficas e de saúde com o uso de medicamentos pelos idosos que responderam ao inquérito postal. Brasil, 2003

\begin{tabular}{|c|c|}
\hline Variáveis & RP (IC95\%) \\
\hline \multicolumn{2}{|l|}{ Faixa etária (anos) } \\
\hline $60-69$ & 1,00 \\
\hline 70 ou mais & $1,07(1,02-1,13)$ \\
\hline \multicolumn{2}{|l|}{ Sexo } \\
\hline Masculino & 1,00 \\
\hline Feminino & $1,11(1,05-1,18)$ \\
\hline \multicolumn{2}{|l|}{ Percepção de saúde } \\
\hline Boa/Muito boa & 1,00 \\
\hline Regular/Ruim/Muito ruim & $1,13(1,04-1,24)$ \\
\hline \multicolumn{2}{|c|}{ Deixar de realizar atividades habituais } \\
\hline Não & 1,00 \\
\hline Sim & $1,09(1,04-1,16)$ \\
\hline \multicolumn{2}{|c|}{ Consultas médicas no último ano } \\
\hline Até 5 & 1,00 \\
\hline 6 ou mais & $1,15(1,10-1,21)$ \\
\hline \multicolumn{2}{|l|}{ Plano privado de saúde } \\
\hline Não & 1,00 \\
\hline $\operatorname{Sim}$ & $1,09(1,04-1,16)$ \\
\hline \multicolumn{2}{|l|}{ Número de doenças } \\
\hline Até 3 & 1,00 \\
\hline 4 ou mais & $1,08(1,02-1,13)$ \\
\hline
\end{tabular}

utilizados $(3,8)$. Esse resultado é semelhante ao observado em outros estudos farmacoepidemiológicos realizados em cidades brasileiras, nos quais a prevalência de uso de medicamentos por idosos tem variado entre $70-92 \%$, com média de utilização entre dois e cinco produtos por pessoa 9,12,13,14,15,20,21. Esse perfil de uso de medicamentos é também comparável aos resultados descritos em países desenvolvidos 22,23,24,25,26. Uma maior utilização de medicamentos pela população idosa é esperada em função da maior ocorrência de doenças crônicas nesta faixa etária, mas seu consumo elevado sugere a contribuição do valor simbólico do medicamento, que propicia a medicalização ${ }^{8}$, bem como a baixa frequência de uso de recursos não farmacológicos para o tratamento de problemas de saúde 20 .

A polifarmácia ocorreu em mais de um terço dos participantes do presente trabalho. O uso de cinco ou mais medicamentos varia bastante tanto entre os estudos nacionais quanto nos internacionais. Entre os estudos nacionais a prevalência de polifarmácia se situa entre 10,9-38\% 5,15,27. Já pesquisas em outros países mostram prevalên- cias ainda mais amplas, entre $11-62 \%$ 22,23,28,29,30. Essas divergências entre as prevalências podem ser em função de aspectos metodológicos, tais como diferenças entre populações-alvo, período recordatório do uso de medicamentos, bem como a definição de polifarmácia. De qualquer forma, o que se constata é a polifarmácia como um fenômeno expressivo, sobretudo entre os idosos mais velhos, fato que evidencia a necessidade de mais estudos para se avaliar a adequação desta prática e o impacto da mesma sobre a saúde e a qualidade de vida dos idosos nas diferentes faixas etárias.

O perfil de utilização de medicamentos dos participantes foi semelhante ao observado em outros estudos nacionais 5,9,13,14,20 e internacionais 22,23,31,32. Essas semelhanças verificadas nos padrões de consumo refletem o perfil das doenças crônicas mais prevalentes entre os idosos.

Observou-se maiores razões de uso de medicamentos por idosos na faixa etária de 70 anos ou mais do que na faixa etária mais jovem, com destaque para os medicamentos que atuam sobre o sistema cardiovascular. Tal fato pode ser explicado, em parte, pelo aumento dos casos de doenças cardiovasculares com a idade, ou ainda pelo cuidado inadequado com a saúde quando mais jovens, resultando na necessidade de farmacoterapia mais complexa em idades mais avançadas. Outros grupos de medicamentos que apresentaram razões do número de medicamentos/idoso acentuadamente altas para essa faixa etária foram os que atuam no sistema nervoso e no trato alimentar/metabolismo. Os principais medicamentos desses grupos terapêuticos têm relação direta com as principais morbidades dos idosos. Entretanto, sua utilização pode também estar relacionada a distorções na prática terapêutica. O vasto arsenal terapêutico disponível no mercado brasileiro, assim como o valor simbólico do medicamento, podem contribuir para o uso excessivo desses produtos, sem que se leve em consideração suas possíveis consequências negativas. Contribui ainda para essa situação a busca da recuperação da saúde por meio dos medicamentos, em detrimento de medidas não farmacológicas. Um exemplo é o uso expressivo de vitaminas e suplementos minerais, cuja relação risco/benefício ainda não é bem estabelecida. Estudo realizado nos Estados Unidos demonstrou que os idosos utilizam multivitaminas e multiminerais com maior frequência que os demais adultos, correndo o risco de usar estes produtos em quantidades superiores às suas necessidades fisiológicas 33. Já uma revisão sistemática da literatura não demonstrou evidências de que o uso de suplementos antioxidantes melhore o prognóstico de diferentes doenças 34 . 
A idade tem se mostrado um importante preditor do uso de medicamentos em vários estudos $9,13,21,22,35$. No presente trabalho, observamos que independentemente da característica avaliada, a prevalência e a média de medicamentos utilizados pelos idosos de maior idade foram sempre superiores àquelas verificadas entre os idosos mais jovens. $\mathrm{Na}$ análise multivariada, idade superior a 70 anos manteve-se associada com o uso de medicamentos no modelo final. Esses achados podem ser parcialmente explicados pelo processo natural de envelhecimento, que acarreta alterações fisiológicas que requerem a utilização de medicamentos. Podem também ser reflexo do modelo assistencial vigente no país, em que a atenção direcionada ao idoso ainda é desintegrada e inefetiva em diversos aspectos. Essa situação favorece a utilização de quantidade cada vez maior de medicamentos nas idades mais avançadas, com vistas a reverter condições negligenciadas ou mesmo mal abordadas por deficiências no processo de atenção à saúde do idoso.

As mulheres utilizaram mais medicamentos que os homens, resultado que está de acordo com outros estudos farmacoepidemiológi$\cos 9,13,15,20,23,35$. Alguns autores apontam que a maior presença de condições crônicas, bem como a maior utilização de serviços de saúde pelas mulheres, podem contribuir para explicar essa maior utilização de medicamentos, já que tais fatores aumentam a probabilidade da prescrição médica 35.

Estudos internacionais apontam que o aumento da utilização de serviços de saúde pelos idosos de maior idade pode ser responsável pelo maior uso de medicamentos 23. Em nosso trabalho a média de medicamentos utilizados foi maior entre os idosos que realizaram mais consultas e entre os que se internaram no último ano. Além disso, o maior número de consultas manteve-se positiva e independentemente associado ao uso de medicamentos.

Neste estudo, os resultados finais da análise multivariada mostraram associação entre uso de medicamentos e piores indicadores de condições de saúde (pior percepção da saúde, maior número de doenças e restrição na realização de atividades habituais). O uso de maior quantidade de medicamentos também foi observado entre os que apresentaram os piores resultados na saúde. Esses achados corroboram os resultados encontrados em outros estudos epidemiológicos nacionais 9,13,14,15 e internacionais 24,35.

A afiliação a algum plano de saúde também se associou de maneira significativa à utilização de medicamentos. Pesquisas em Bambuí e em Belo Horizonte também evidenciaram associação entre afiliação a plano de saúde e uso de medicamentos 13,14. Acredita-se que a filiação ao plano de saúde pode aumentar a possibilidade de uso de medicamentos por facilitar o acesso a mais médicos 14, resultando em um maior número de prescrições.

Os resultados obtidos por meio deste inquérito postal ampliam o conhecimento a respeito da utilização de medicamentos pelos idosos brasileiros, evidenciando a necessidade de aprimoramento da assistência farmacêutica voltada para esse subgrupo da população, mais vulnerável ao risco de iatrogenia.

O uso de maior quantidade de medicamentos pelos idosos mais velhos que foi observado neste trabalho, embora muitas vezes necessário, pode acarretar graves consequências, tais como maior número de reações adversas, risco de uso de medicamentos inadequados, dificuldade de adesão ao tratamento farmacológico, além de levar ao incremento do risco de morbidades e mortalidade. Outros fatores podem contribuir para a iatrogenia, como a utilização de medicamentos de eficácia e segurança questionáveis. Acrescentem-se as limitações físicas e cognitivas presentes nesta etapa da vida que podem dificultar a correta utilização desses produtos, especialmente em situações de polifarmácia. A partir do conhecimento dos fatores que se mostraram associados ao uso de medicamentos, espera-se contribuir para a elaboração de políticas públicas direcionadas ao bem-estar desse subgrupo populacional, que visem à adequação da assistência farmacêutica às suas reais necessidades, promovendo desta forma a racionalização do uso de medicamentos e, consequentemente, a otimização da terapêutica medicamentosa. 


\section{Resumo}

O objetivo deste estudo foi avaliar fatores associados ao uso de medicamentos por idosos. Foi realizado um inquérito postal nacional, com 3 mil idosos, selecionados com base no cadastro do Instituto Nacional do Seguro Social. Foram estimadas a prevalência e a média de medicamentos utilizados nos 15 dias anteriores à pesquisa, segundo faixas etárias. A prevalência de uso de medicamentos foi de $83 \%$, sendo de $87,3 \%$ no grupo de 70 anos ou mais, e de 78,8\% no de 60-69 anos $(p<0,05)$. Os idosos com 70 anos ou mais usaram em média 4,4 medicamentos, os mais jovens 3,3. Os medicamentos mais utilizados pertenciam ao sistema cardiovascular. Idade de 70 ou mais anos, sexo feminino, pior percepção de saúde, interrupção de atividades habituais, mais de 6 consultas médicas no último ano, filiação a plano de saúde e relato de 4 ou mais doenças estavam associados ao uso de medicamentos $(p<0,05)$ entre os participantes. Os resultados ampliam o conhecimento a respeito da utilização de medicamentos pelos idosos brasileiros, evidenciando a necessidade de aprimoramento da assistência farmacêutica voltada para esse subgrupo da população.

Farmacoepidemiologia; Polimedicação; Uso de Medicamentos; Idoso

\section{Colaboradores}

A. L. Silva participou da organização do banco de dados, do desenvolvimento, análise e interpretação dos dados, revisão da literatura e redação do artigo final. A. Q. Ribeiro participou do planejamento e supervisão da coleta de dados, organização do banco de dados, análise e interpretação dos dados e revisão da versão final do manuscrito. C. H. Klein participou da elaboração do projeto, análise dos resultados e revisão do artigo final. F. A. Acurcio colaborou na elaboração e orientação do projeto, planejamento da coleta de dados, análise dos resultados e revisão do artigo final.

\section{Agradecimentos}

À Confederação Brasileira dos Aposentados e Pensionistas (COBAP). Aos integrantes do Grupo de Pesquisa em Farmacoepidemiologia da Faculdade de Farmácia da UFMG. Ao Ministério da Saúde do Brasil/Fundo Nacional de Saúde, por intermédio da então Gerência Técnica de Assistência Farmacêutica (GTAF/DAB/SPS convênio $n^{\circ}$. 796/2002), pelo financiamento. À Faculdade de Farmácia da UFMG e à Fundação Oswaldo Cruz, pelo apoio logístico. À CAPES pelo fomento de bolsa de mestrado a A. L. Silva.

\section{Referências}

1. Instituto Brasileiro de Geografia e Estatística. Síntese de indicadores sociais 2008 - uma análise das condições de vida da população brasileira. Rio de Janeiro: Instituto Brasileiro de Geografia e Estatística; 2008. (Estudos e Pesquisas: Informação Demográfica e Socioeconômica, 23).

2. Instituto Brasileiro de Geografia e Estatística. Projeção da população do Brasil por sexo e idade 1980-2050 - revisão 2008. v. 24. Rio de Janeiro: Instituto Brasileiro de Geografia e Estatística; 2008.

3. Instituto Brasileiro de Geografia e Estatística. Tábuas completas de mortalidade 2005. http://www. ibge.gov.br (acessado em 30/Abr/2008).

4. Veras R. Envelhecimento populacional contemporâneo: demandas, desafios e inovações. Rev Saúde Pública 2009; 43:548-54.
5. Mosegui GBG, Rozenfeid S, Veras RP, Vianna CMM. Avaliação da qualidade do uso de medicamentos em idosos. Rev Saúde Pública 1999; 33:437-44.

6. Ribeiro AQ, Acurcio FA, Wick JY. Pharmacoepidemiology of the elderly in Brazil: state of the art. Consult Pharm 2009; 24:30-44.

7. Rozenfeld S. Prevalência, fatores associados a mau uso de medicamentos entre idosos: uma revisão. Cad Saúde Pública 2003; 19:717-24.

8. Lefévre F. A função simbólica dos medicamentos. Rev Saúde Pública 1983; 17:500-3.

9. Coelho Filho JM, Marcopito LF, Castelo A. Perfil de utilização de medicamentos por idosos em área urbana do Nordeste do Brasil. Rev Saúde Pública 2004; 38:557-64. 
10. Lima MG, Ribeiro AQ, Acurcio FA, Rozenfeld S, Klein CH. Composição dos gastos privados com medicamentos utilizados por aposentados e pensionistas com idade igual ou superior a 60 anos em Belo Horizonte, Minas Gerais, Brasil. Cad Saúde Pública 2007; 23:1423-30.

11. Lima-Costa MF, Barreto SM, Firmo JO, Uchoa E. Socioeconomic position and health in a population of Brazilian elderly: the Bambuí Health and Aging Study (BHAS). Rev Panam Salud Pública 2003; 13:387-94.

12. Lebrão ML, Laurenti R. Saúde, bem-estar e envelhecimento: o estudo SABE no Município de São Paulo. Rev Bras Epidemiol 2005; 18:127-41.

13. Loyola Filho AI, Uchoa E, Lima-Costa MF. Estudo de base populacional sobre o consumo de medicamentos entre idosos: Projeto Bambuí. Cad Saúde Pública 2005; 21:545-53.

14. Ribeiro AQ, Rozenfeld S, Klein CH, César CC, Acurcio FA. Inquérito sobre uso de medicamentos por idosos aposentados, Belo Horizonte, MG. Rev Saúde Pública 2008; 42:724-32.

15. Rozenfeld S, Fonseca MJM, Acurcio FA. Drug utilization and polypharmacy among the elderly: a survey in Rio de Janeiro City, Brazil. Rev Panam Salud Pública 2008; 23:34-43.

16. Acurcio FA, Silva AL, Ribeiro AQ, Rocha NP, Silveira MR, Klein $\mathrm{CH}$, et al. Complexidade do esquema terapêutico prescrito para idosos, Belo Horizonte/ MG. Rev Assoc Med Bras 2009; 55:468-73.

17. Acurcio FA, Rozenfeld S, Ribeiro AQ, Klein $\mathrm{CH}$, Moura CS, Andrade CR. Utilização de medicamentos por aposentados brasileiros. 1 - Metodologia e resultados de cobertura de inquérito multicêntrico. Cad Saúde Pública 2006; 22:87-96.

18. Melo JMS, editor. DEF 2002-2003: Dicionário de especialidades farmacêuticas. 31a Ed. Rio de Janeiro: Publicações Científicas; 2003.

19. World Health Organization. Anatomical therapeutic chemical (ATC) classification index with defined daily doses (DDD's). Oslo: World Health Organization; 2000.

20. Flores LM, Mengue SS. Uso de medicamentos por idosos em região do sul do Brasil. Rev Saúde Pública 2005; 39:924-9.

21. Loyola Filho AI, Uchoa E, Lima-Costa MF. Estudo epidemiológico de base populacional sobre uso de medicamentos entre idosos na Região Metropolitana de Belo Horizonte, Minas Gerais, Brasil. Cad Saúde Pública 2006; 22:2657-67.

22. Chen YF, Dewey ME, Avery AJ. Self-reported medication use for older people in England and Wales. J Clin Pharm Ther 2001; 26:129-40.
23. Linjakumpu T, Hartikainen S, Klaukka T, Veijola J, Kivelä SL, Isoaho R. Use of medications and polypharmacy are increasing among the elderly. J Clin Epidemiol 2002; 55:809-17.

24. Fuchs Z, Novikov I, Blumstein T, Chetrit A, Gindin J, Modan B. Patterns of drug use among the community-dwelling old-old population in Israel. Isr Med Assoc J 2003; 5:346-51.

25. Gallagher PF, Barry PJ, Ryan C, Hartigan I, O'Mahony D. Inappropriate prescribing in an acutely ill population of elderly patients as determined by Beers' Criteria. Age Ageing 2008; 37:96101.

26. Ramage-Morin PL. Medication use among senior Canadians. Health Rep 2009; 20:37-44.

27. Almeida OP, Ratto L, Garrido R, Tamai S. Fatores preditores e conseqüência clínica do uso de múltiplas medicações entre idosos atendidos em um serviço ambulatorial de saúde mental. Rev Bras Psiquiatr 1999; 21:152-7.

28. Junius-Walker U, Theile G, Hummers-Pradier E. Prevalence and predictors of polypharmacy among older primary care patients in Germany. Fam Pract 2007; 24:14-9.

29. Salazar JA, Poon I, Nair M. Clinical consequences of polypharmacy in elderly: expect the unexpected, think the unthinkable. Expert Opin Drug Saf 2007; 6:695-704.

30. Kuijpers MA, van Marum RJ, Egberts AC, Jansen PA. OLDY (OLd people Drugs \& dYsregulations) Study Group. Relationship between polypharmacy and underprescribing. Br J Clin Pharmacol 2008; 65:130-3.

31. Steinman MA, Landefeld CS, Rosenthal GE, Berthenthal D, Sen S, Kaboli PJ. Polypharmacy and prescribing quality in older people. J Am Geriatr Soc 2006; 54:1516-23.

32. Wawruch M, Zikavska M, Wsolova L, Kuzelova M, Tisonova J, Gajdosik J, et al. Polypharmacy in elderly hospitalised patients in Slovakia. Pharm World Sci 2008; 30:235-42.

33. Rock CL. Multivitamin-multimineral supplements: who uses them? Am J Clin Nutr 2007; 85:277S-9S.

34. Bjelakovic G, Nikolova D, Gluud LL, Simonetti RG, Gluud C. Antioxidant supplements for prevention of mortality in healthy participants and patients with various diseases. Cochrane Database Syst Rev 2008; 16(2):CD007176.

35. Woo J, Ho SC, Yuen YK, Lau J. Drugs use in an elderly Chinese population: prevalence and associated factors. Gerontology 1995; 41:98-108.

Recebido em 22/Nov/2010

Versão final reapresentada em 22/Jul/2011

Aprovado em 06/Fev/2012 